\title{
EHE, EHESCHEIDUNG UND WIEDEREHE IN DER ORTHODOXEN KIRCHE
}

\author{
Irimie Marga*
}

\begin{abstract}
Man, entirely, can only be understood as "together". From this "together", part of man, family was born, as the first and unchangeable expression of communion. The family is grounded on Pentecost, namely through the Sacrament of Holy Matrimony, that is why it can be also named as "the little Church Pentecost". We confess that the Church is One, Holy Catholic and Apostolic, but when we state these we do not only refer to the Great Church but also to the Little Church. The Orthodox Church teaches that the Sacrament of Holy Matrimony is a Sacrament not to be dissolved, its indissolubility is not static, belonging to the past and that would constraint up to asphyxia, but dynamic, vivid, a target that attracts and that is to be reached through spiritual fight sustained by the grace given in the Sacrament of Holy Matrimony. Divorce is a sin and a tragedy. It exists, it cannot be denied and it cannot be interdicted either. Through divorce, indissolubility is not cancelled, but only abandoned. The Orthodox Church does not accept divorce as it does not accept sin, but only allows, tolerates or recognizes divorce, as undeniable painful reality. According to the principle of iconomy, the Church has chosen between two unwanted situations: either to reject the divorced spouses, as having missed Salvation, or reintegrate them in the Church by tolerating, allowing a new marriage. Between the two, the Church has chosen the smallest evil, namely, tolerating the divorced to remarry.
\end{abstract}

Keywords: Family, Church, marriage, matrimony, sacrament, grace, love, failure, divorce, toleration, salvation, remarriage.

*PhD, Associate Professor, "St. Andrei Şaguna"Faculty of Orthodox Theology, at "Lucian Blaga" University, Sibiu, Romania.Last work:Irimie Marga, Gerald G. Sander, Dan Sandu (eds.), Religion zwischen Kirche, Staat und Gesellschaft - Religion between Church, State and Society,Hamburg,Verlag Dr. Kovač, 2007, 288 p. Contact:irimiemarga@yahoo.de 


\section{Einführung}

Das Bild der Dreifaltigkeit im Menschen sieht man davon, dass der volle Mensch nicht alleine geschaffen wurde, sondern Mann und Frau, in einer wesentlichen, ontologischen Kommunio. Der Mensch, in seiner Vollendung, kann man nicht alleine verstehen, sondern nur ,zusammen"1.

Aus diesem konstitutiven „Zusammen-sein” ist die Familie entstanden, als primäre und inalienabel Verwirklichung der Kommunion. Die Familie hat eine besondere Bedeutung in der ganzen Heilsgeschichte: in der Familie war der Sündenfall, durch die Familie vermehrt sich die Menschheit, von der Familie kommt die Erbsünde, in der Familie beginnt auch die Wiederherstellung der verderbten Kommunion. Aus diesem Grund nennt der Hl.Ap. Paulus die Familie als „ecclesia domestica” (Röm. 16, 5), und die Hl. Kirchenväter als „ecclesia minor" (Johannes Chrisostomos), die auf die Basis der „ecclesia maior” steht. Die beiden „ecclesia” befinden sich in Kontinuität und unzerstörbare Verbindung.

Durch das Herabkommen des Heiligen Geistes wird die grosse Kirche gegründet, als Heilsort durch Christus, in der Liebe des Gott-Vaters. Die kleine Kirche, die Familie, gründet sich durch ein Herabkommen des Heiligen Geistes auch, nähmlich durch das Ehesakrament, deshalb kann man das Ehesakrament als „kleines Pfingsten"in,,ecclesia minor" nennen.

Das Ehesakrament ist also ein neues Pentekostas, ein Herabkommen der Gnade Gottes über das Ehepaar, als Antvort auf die gegenseitige Liebe der Partner, dadurch wird die christliche Familie gegründet, wo die Gnade die Ehepartner in einem neuem Leben vereinigt und das Vorkosten des ewigen Lebens schenkt. Wie die Familie die Zelle der Gesellschaft ist, so ist auch die kleine Kirche die Zelle der grossen Kirche, die sich in einer engen gegenseitigen Verbindung befinden.

1 Dumitru Stăniloae, Teologia Dogmatică Ortodoxă, III-e.Band, Bukarest, 1978, S.180. 
Die durch das Ehesakrament verwirklichte Einheit ist keine Einheit aus der niedrigen Welt, sondern eine Einheit von oben ${ }^{2}$. Sie ist eine Einheit, die von dem Hl. Geist eine Vocation und eine Mission bekommt, das heisst, das die Ehe kein statisches Akt ist. Auf einer Seite ist das Ehesakrament ein Akt der Schenkung der Gnade, als göttliche Energie, auf die andere Seite ist dieses Sakrament ein kontinuierlichen Akt des Wachsens der Ehepartner, in Liebe und gegenseitige Opferung. Ohne dieser göttlichen Gnade fällt die Familie in einem einfachen, biologischen Bezug, der oft zur Langweile und Zerstörung führt. Die Gnade ernährt und hält die Liebe der Ehepartner in einen lebendigen, aktiven und unendlichen Zustand.

Durch die Liebe und gegenseitige Schenkung halten die Ehepartner, mit der Unterstützung des Hl. Geistes, die Einheit in und durch Christus. In diesem Kontext entwickelt sich auch die erotische Liebe, die die Ehepartner zu einem Leib vereinigt (Mat. 19, 6). Die Kirche unterschätzt nicht die leibliche Vereinigung zwischen einen Mann und eine Frau, die eine dopplete Rolle spielt, die Erfüllung der sensuellen Neigungen und die Procreation. Die beiden Aspekte schliessen sich nicht gegeseitig aus, sondern sie befinden sich in einer verantwortliche Abhängigkeit ${ }^{3}$.

\section{Das Ehesacrament als „kleines Pfingsten” in „ecclesia minor"}

Der Vergleich zwischen der grossen Kirche und kleinen Kirche kennt viele andere Aspekte, zum Beispiel, dem Glaubensbekenntnis in der grossen Kirche entspricht der Treue der Ehepartner in der kleinen Kirche, oder die Eigenschaften der grossen Kirche kann man in den Eigenschaften der kleinen Kirche erkennen. Wir bekennen, dass die Kirche eine, heilige, katholische und

\footnotetext{
${ }^{2}$ Ilie Moldovan, ,, In Hristos şi în Biserică”. Adevărul şi frumuseţea căsătoriei. Teologia iubirii,II-e Band,Alba Iulia, 1996, S.22-23.

3 William Basil Zion, Eros şi transfigurare,Alba Iulia, ReîntregireaVerlag, 2001, 303S.
} 
apostolische Kirche ist. So verstehen wir auch die kleine Kirche, die Familie.

Die Familie ist eine, im Sinne, dass sie sich einmal für immer, für das ganze Leben schliesst, oder dass die Ehe unauflösbar und monogamisch ist. Das Ehesakrament vereinigt das Ehepaar in einer Einheit des Lebens in einem einzigen Christus. Die Gnade des Ehesakraments verstärkt, unter sich und mit Christus, die Einheit der Partner und verteidigt die Aufgabe der Unauflösigkeit der Ehe, genau so wie die Pfingsten alle Gläubigen in einer Kommunio vereinigt hat.

Die Familie ist heilig durch ihre Aufgabe, dass die Ehepartner, durch das Zusammenleben, die Heiligkeit erreichen können. Der Ehemann und die Ehefrau sind nicht heilig in sich, aber sie wollen die Heiligkeit, mit der Unterstützung des Hl. Geistes, gewinnen. Der Ausgangspunkt der Heiligkeit für alle Menschen ist das Pfingsten, so ist es auch das Ehesakrament für die Ehepartner: der Ausgangspunkt der Heiligkeit.

Die Familie ist katholisch, weil sie die ganze Menschheit betrifft, dadurch vermehrt sich die ganze Menschheit. Das Pfingsten hat die Gnade über das ganze Cosmos gegossen, so das Ehesakrament bezieht sich auf alle Menschen.

Die Familie ist apostolisch, im Sinne, dass das Ehesakrament von Christus selbst gegründet wurde und durch die apostolische Sukzession bis heute vermittelet ist. Die wahre Sakramentalität entdeckt sich in der ununterbrochene apostolische Kontinuität, die das Pfingsten mit jedem Ehesakrament verbindet.

Die Gründung der kleinen Kirche durch das Herabkommen der Gnade Gottes über jede neue Familie („,das kleine Pfingsten”), als göttliche Antwort auf die gegenseitige Liebe der Ehepartner ${ }^{4}$, offenbart uns die theologische Bedeutung des Ehesakraments, die weitweg von allen juridischen Konzepte über die Ehe ist. Aus diesem Grund hat die Orthodoxe Kirche die lateinischen juridischen Sprüche

\footnotetext{
${ }^{4}$ Paul Evdokimov, Taina iubirii. Sfinţenia unirii conjugale în lumina tradiţiei ortodoxe,übersetzung Gabriela Moldoveanu, überprüfung und Übersetzung verbessern Vasile Răducă, Bukarest, Christiana christliche Ärztekammer, 1994, 261S.
} 
über die Familie nicht übernommen ${ }^{5}$, wie zum Beispiel „consensus facit nuptias"u.a.

Das Ehesakrament ist ein heiliger Akt, der die Kirche in der Mitte der Familie bringt und, umgekehrt, der die Familie in der Kirche eingliedert. Die Gnade des Hl. Geistes, die in dem Ehesakrament einberufen wird, unterstützt und verteidigt diese doppelte Bewegung.

\section{Die Unauflösigkeit der Ehe}

Die Orthodoxe Kirche lehrt, dass die Ehe ein unauflösbares Sakrament ist. Die Unauflösigkeit der Ehe ist praktisch keine Tatsache, die schon durch das Sakrament verwirklicht ist, sondern eine Eigenschaft, die das ganze Leben bewahrt und erfüllt wird; dafür bekommt man eine spezielle Gnade, als Hilfe und Unterstützung, die uns nicht pasiv lässt, sondern die aktiv unter der Partner entwickeln muss. So gesehen ist die Unauflösigkeit nicht eine gegebene Sache, sondern ein Werk des Willens für die beiden Ehepartner ${ }^{6}$.

Nach der Lehre der Hl. Väter der Kirche ist das Ehesakrament nicht ein einfaches Akt, das man wie einen Vertrag schliesst, sondern die Ehe ist ein kontinuierliches Wachsen der Partner, in gegenseitige Liebe und Verantwortung, in Unterstützung und Rettung aus allen kritischen Situationen, mit der Hilfe und Mitwirken (Synergie) der Ehegnade ${ }^{7}$.

So muss man auch die Unauflösigkeit der Ehe verstehen, nicht als eine statische Tatsache, die schon in Vergangenheit entschieden ist, oder die bis zur Erstickung zwingt, sondern als eine dinamische und lebendige Wirkung, als ein Zweck, der an sich zieht,

${ }^{5}$ Irimie Marga, Actualitatea sfintelor canoane,in: „Revista Teologică”, Yahre XII (84),Nr. 3, Juli-September, 2002, S.41-46.

${ }^{6}$ Dumitru Stăniloae, op.cit.,III-e.Band, S.185.

${ }^{7}$ John Meyendorf, Căsătoria: o perspectivă ortodoxă,übersetzung Cezar Login, Cluj-Napoca, PatmosVerlag, 2007, S.20f. 
als einen Ziel, der durch einen entsprechenden geistigen Kampf erreichen wird.

Der grösste von Gott bekommene Geschenk des Menschens ist die Freiheit, deshalb darf die Kirche nie die Freiheit des Menschens begrezen, sei es auch wann die Freiheit in Richtung Sünde oder Entfremdung von Gott geht. Desto mehr darf die Unauflösigkeit, als wesentliches Merkmal der Ehe, nicht die Freiheit ersticken, sei es auch wenn die Freiheit zum Scheitern führt ${ }^{8}$.

Gott hat uns eine volle Freiheit verschenkt, einschliesslich die Freiheit den Gott zu verneinen, die sowieso die Existenz Gottes nicht beinflüssen kann. Die Unauflösigkeit der Ehe existiert in dieser vollen Freiheit, ausser jedem Zwang, in einer Freiheit der Partner, der auch in der Richtung der Ehescheidung gehen darf, ohne damit die Unauflösigkeit, als Ziel und Merkmal der Ehe, abzuschaffen. Die geschiedenen Partner erkennen mit Schmerz und Bedauern den Scheitern und die Sünde, ohne damit die Unauflösigkeit der wahren Ehe, die sie nicht erreichen konnten, zu bestreiten. Die Menschen, die den Everest nicht errobern, können nicht den Everest verneinen. Anders gesagt, ist die Unauflösigkeit eine Vocatio, eine Aufgabe, und nicht eine gezwungene Schlussfolgerung der Eheschliessung. Eine gezwungene Unauflösigkeit führt die Vocatio in einer Suffocatio der Ehe (Erstickung).

Die Unauflösigkeit der kleinen Kirche kann man auch mit der Heiligkeit der grossen Kirche vergleichen: die grosse Kirche ist heilig durch ihren Ziel, obwohl die Mitglieder sündige Personen sind, die man zur Heiligkeit nicht erzwingen kann. Die kleine Kirche ist unauflösbar durch ihre Aufgabe, die den sündigen Willen der Partner nicht erzwingen kann. Die Unauflösigkeit der Ehe ist wert, wenn sie nur in voller Freiheit angenommen wird.

${ }^{8}$ Grigorios Larentzakis, Die Orthodoxe Kirche. Ihr Leben und ihr Glaube, Graz, Styria Verlag, 2000, S.78f. 


\section{Die Ehescheidung}

Die Ehescheidung war und ist eine schmerzhafte Tatsache des menschlichen Lebens. Niemand kann die unerwünschte Anwesenheit der Ehescheidung in dieser Welt verneinen.

Die Ehescheidung ist eine Sünde und eine Tragödie. Niemand liebt die Ehescheidung, niemand wählt freiwillig die Ehescheidung, niemand verteidigt sie. Die Ehescheidung gehört volens-nolens unserer Existenz, deshalb kann man sie nicht ignorieren und, leider, nicht verbieten. Man kann die freiwillige angenommene Sünde moralisch verbieten, aber wir können nicht die unvorgesehene und unerwünschte Unfälle verbieten. Das Verbieten der Ehescheidung ist unlogisch, ist ein Non-sens, ein Unsinn, ist wie das Verbieten der Erdbeben, der Überschwemungen, der Krankheit usw...

Eine gesunde Ehe ist unauflösbar. Die grösste Krankheit der Familie ist die Ehescheidung, dadurch wird die Unauflösigkeit nicht annulliert, sondern verlassen. Die Sünden der Gläubigen können die Heiligkeit der Kirche nicht annullieren, sondern die Sünden verstärken das Schmerzen einer nicht erfüllten Heiligkeit.

Jeder Arzt liebt die Gesundheit und hat die Krankheit nich gern, das heisst nicht, dass der Arzt die Krankheit verbieten kann. Es wäre ein Unsinn. Aus diesem Grund kann die Kirche nicht unsinnig sein, die Ehescheidung zu verbieten. Die Kirche kann nur aufmerksam machen, die Krankheit der Ehescheidung verbeugen oder behandeln. Die Kirche kann die Ehepartner geistlich beraten, mit der Gnade $\mathrm{zu}$ arbeiten in einer Synergia, in einer verantwortlichen und opferischen Liebe, um jeden Unfall zu verbeugen ${ }^{9}$.

Einige Theologen haben gesagt - nicht selten - dass die Orthodoxe Kirche die Ehescheidung akzeptiert. Nein! Die Orthodoxe

9 Ioan N. Floca, Încetarea şi desfacerea căsătoriei civile şi a cununiei religioase sau divorţul în lumina învăţăturii creştine,in: „Mitropolia Ardealului”, Yahre XIX, Nr. 10-12, Oktober-Dezember,1974, S.572-579. 
Kirche akzeptiert die Ehescheidung nicht, sie kann die Sünden nicht akzeptieren, aber sie muss die Ehescheidung tolerieren und anerkennen, als eine schmerzhafte, unbestreitbare Wirklichkeit. Das Heilen einer Krankheit beginnt nicht mit einer gleichgültigen Akzeptierung, sondern mit ihrer Anerkennung, in Mitleid mit den Kranken, um die richtige Behandlung $\mathrm{zu}$ finden. So kann man verstehen, warum die Orthodoxe Kirche mit der Ehescheidung nicht einverstanden ist, sondern sie kämpft gegen dieser Tragödie, aber sie verurteilt die Geschiedenen nicht, sondern sie liebt die, die sich in einer Notsituation befinden, um auch eine Notlösung zu finden.

Andere Theologen haben geglaubt, dass die Orthodoxe Kirche die Ehepartner scheidet, oder dass sie das Ehesakrament entbinden kann. Wieder falsch! Die Orthodoxe Kirche kann nicht ein Sakrament auflösen, kann nicht auseinandernmachen, was Gott vereinigt hat. Das ist eine Unmöglichkeit, zum Beispiel die Kirche kann die Euharistie nicht mehr wieder in Brot und Wein umwandeln. Die Kirche kann nicht etwas ,umgekehrt" machen, oder die Sakramente wieder ,umdrehen”. Nur die Empfänger der Sakramente - nicht die Kirche! - können gegen der Gnade wirken, wenn sie den Glauben verlieren. Aus diesem Grund kennt die Orthodoxe Kirche die Praxis einer „Anullierung” eines Sakramentes gar nicht, auch die sogenannte ,Anullierung der Ehe" ist fremd für uns ${ }^{10}$.

Die Kirche kann nur eines: sie anerkennt die zerstörbare Wirklichkeit der Sünden, um etwas für die Sünder zu machen. Die sogennante ,kirchliche Ehescheidung" ist also eine notwendige Feststellung, hat einen formällen und pasiven Charakter und, auf keinen Fall, eine liturgisch-sakramentale Dimension.

Die in einer unerwünschten Kriese gefallene Ehepartner können die gegenseitige Liebe verlieren, das Mitwirken (Synergia) mit der Ehegnade aufhörren, und so in einer Ehescheidung zu kommen, aber sie können nicht die Unauflösigkeit der Ehe zerstörren. Die Ehegnade bleibt in eine schmerzhafte Wartung, in

10 Timotei Seviciu, Divorţul şi Biserica Romano-Catolică,in: „Mitropolia Banatului”, Yahre XXI, Nr. 7-9, Juli-September, 1971, S.487-490. 
einer Unwirksamkeit, weil die Ehepartner nicht mehr aktiv sind. Diesen Zustand ist ähnlich mit der Ikone Christi, der an einer Tür klopft, aber die Tür öffnet sich nicht. Das ist das grosse Drama der Ehescheidung.

\section{Das Wiederheiraten}

Die Tragödie der geschiedenen Ehepartner nach einer unglücklichen Ehe stellt der Kirche eine schwierige Frage: was macht die Kirche in solcher Situationen, wie kann sie für das Heil der beiden helfen?

Vom Anfang an muss man sagen, dass die Tragödie der geschiedenen Ehepartner auch eine Tragödie der Kirche ist. In diesem Fall darf die Kirche nicht unwirksam oder unveränderlich bleiben. Die Antwort auf die erwähnte Frage finden wir in der Praxis der ungeteillte Frühkirche.

Gemäss dem Prinzip der Oikonomia (Nachlass) hat die Kirche zwischen zwei unerwünschte Situationen gewählt: entweder die Geschiedenen, als grössten Sündiger, zu ablehnen, oder die Geschiedenen $\mathrm{zu}$ tolerieren und ihnen eine neue Chance $\mathrm{zu}$ gewähren. Aus diese zwei Varianten hat die Kirche, vom Anfang an, den kleineren Übel gewählt, also die Tolerierung der Wiederheratung ${ }^{11}$.

Die erste Situation, der grössere Übel, könnte die Kirche nicht annehmen, weil die Kirche die Heilstür für niemanden zusperren darf. Anders gesagt, in der Kirche darf man nie sagen: „Es gibt keine Chance mehr!". So was kann man nur in einem Gefängniss hörren, wo die Freiheit völlig begrenzt ist.

In Notsituationen muss die Kirche neue Lösungen finden, nicht als neue Regelungen, sondern als Ausnahmen, als „excepta” (Mat. 5, 32), wie der Christus selbst gesagt hat. Die Worte Christi

${ }^{11}$ Liviu Stan, Despre principiile canonice fundamentale ale Ortodoxiei,in: „Biserica şi Dreptul. Studii de Drept canonic ortodox”, III-e. Band, Sibiu, Andreiana Verlag, 2012, S.5-24. 
„ausser Ehebruch" muss man nicht eng verstehen, sondern in einem breiterem Sinn. Das „Ehebruch” im biblischen Sinn bedeutet einen spirituellen Tod, in dem alle Geschiedenen fallen.

Eine Ausnahme kann man einmal akzeptiert werden, höchstens zweimal, sonst die unbegrenzte Wiederholung führt zu einem neuen Gesetzt, dem gegen dem ersten ist. Deshalb ist eine Wiederheiratung möglich, höchstens die zweite, aber nicht mehr, um nicht $\mathrm{zu}$ einer unkontrollierte Wiederholung zu führen. Das ist der kanonisch-theologischen Sinn der Synode aus Konstantinopel, vom Jahre 920, der die Tetragamie verboten hat ${ }^{12}$.

Der Prinzip der Oikonomia ist kein neues Gesetzt der Kirche, es ist ein Notweg für die, die neue Möglichkeiten brauchen, um das Heil nicht zu verlieren. Die Ausnahme zerreist das Gesetzt nicht, sondern schliest die Erstickung der Freiheit aus, wann die Buchstabe umbringt. Deshalb kann man das Prinzip der Oikonomia als Prinzip der verantwortliche Freiheit nennen.

Das Prinzip der Oikonomia finden wir bei den H1. Väter und in der ganzen Kirchengeschichte, besonders im Kanon 8 von der ersten ökumenischen Synode, wo die Cataren, die Puritanisten, die Sauberer, von der Kirche weggeschmiessen wurden. Die Kirche darf nicht exclusivistisch sein, sondern barmherzig im Not $^{13}$.

Man muss auch sagen, dass das Prinzip der Oikonomia kein Favor, lateinisch gesagt kein „Privilegium” ist, deshalb hat die Ostkirche dieses Wort aus dem römischen Recht nicht übernommen ${ }^{14}$. In der Kirche darf niemand favorisiert sein, deshalb ist die zweite Ehe kein Favor, sondern ein Notschiff für gescheiterte Partner.

12 Iorgu D. Ivan, Recăsătorirea soților despărţiţi. Studiu de drept canonic,Bukarest, 1937, 95S.

${ }^{13}$ Vlassios I. Phidas, Drept Canonic. O perspectivă ortodoxă,Iaşi, Trinitas Verlag, 2008, 214S.

${ }^{14}$ Ioan Tamaş, Drept matrimonial catolic,Iaşi „Presa Bună” Verlag, 1994, 199S. 


\section{Schlussfolgerungen}

Als Schlussfolgerungen, kann man sagen, dass die durch das Ehesakrament verwirklichte Einheit, keine Einheit aus der niedrigen Welt, sondern eine Einheit von oben ist $^{15}$. Sie ist eine Einheit, die von dem Hl. Geist eine Vocation und eine Mission bekommt, das heisst, das die Ehe kein statisches Akt ist. Auf einer Seite ist das Ehesakrament ein Akt der Schenkung der Gnade, als göttliche Energie, auf die andere Seite ist dieses Sakrament ein kontinuierlichen Akt des Wachsens der Ehepartner, in Liebe und gegenseitige Opferung.

Die Orthodoxe Kirche lehrt, dass die Ehe ein unauflösbares Sakrament ist. Die Unauflösigkeit der Ehe ist praktisch keine Tatsache, die schon durch das Sakrament verwirklicht ist, sondern eine Eigenschaft, die das ganze Leben bewahrt und erfüllt wird.

Die Ehescheidung ist eine Sünde und eine Tragödie.Eine gesunde Ehe ist unauflösbar. Die grösste Krankheit der Familie ist die Ehescheidung, dadurch wird die Unauflösigkeit nicht annulliert, sondern verlassen.

Gemäss dem Prinzip der Oikonomia (Nachlass) hat die Kirche zwischen zwei unerwünschte Situationen gewählt: entweder die Geschiedenen, als grössten Sündiger, zu ablehnen, oder die Geschiedenen $\mathrm{zu}$ tolerieren und ihnen eine neue Chance zu gewähren.Die zweite Ehe ist ein barmherziges Zeichen für die geschiedenen Partner, um das Heil nicht zu verlieren. Der liebe Gott widerspricht sich nicht, in seiner Heiligkeit, wenn Er den Sünder vergibt und eine neue Chance gewährt.

${ }^{15}$ Ilie Moldovan, ,,In Hristos şi în Biserică ”. Adevărul şi frumuseţea căsătoriei. Teologia iubirii,II-e Band,Alba Iulia, 1996, S. 22-23. 


\section{Bibliographie}

1. Basil Zion,William, Eros şi transfigurare,Alba Iulia, Reîntregirea Verlag, 2001.

2. Evdokimov,Paul, Taina iubirii. Sfinţenia unirii conjugale în lumina tradiţiei ortodoxe,übersetzung Gabriela Moldoveanu, überprüfung und Übersetzung verbessern Vasile Răducă, Bukarest, Christiana christliche Ärztekammer, 1994.

3. Floca,Ioan N., Încetarea şi desfacerea căsătoriei civile şi a cununiei religioase sau divorţul în lumina învăţăturii creştine,in: „Mitropolia Ardealului”, Yahre XIX, Nr. 10-12, Oktober-Dezember, 1974, S. 572-579.

4. Ivan,Iorgu D., Recăsătorirea soţilor despărţiţi. Studiu de drept canonic,Bukarest, 1937.

5. Larentzakis,Grigorios, Die Orthodoxe Kirche. Ihr Leben und ihr Glaube, Graz, Styria Verlag, 2000.

6. Marga,Irimie, Actualitatea sfintelor canoane,in: „Revista Teologică”, Yahre XII (84), Nr. 3, Juli-September, 2002, S. 41-46.

7. Meyendorf,John, Căsătoria: o perspectivă ortodoxă,übersetzung Cezar Login, Cluj-Napoca, Patmos Verlag, 2007.

8. Moldovan,Ilie, ,In Hristos şi în Biserică”.'Adevărul şi frumuseţea căsătoriei.

Teologia iubirii,II-e Band,Alba Iulia, 1996.

9. Phidas,Vlassios I., Drept Canonic. O perspectivă ortodoxă,Iaşi, Trinitas Verlag, 2008, 214S.

10. Seviciu,Timotei, Divorţul şi Biserica Romano-Catolică,in: „Mitropolia Banatului", Yahre XXI, Nr. 7-9, Juli-September, 1971, S. 487-490.

11. Stan,Liviu, Despre principiile canonice fundamentale ale Ortodoxiei,in: „Biserica şi Dreptul. Studii de Drept canonic ortodox”, III-e. Band, Sibiu, Andreiana Verlag, 2012, S. 5-24.

12. Stăniloae,Dumitru, Teologia Dogmatică Ortodoxă, III-e. Band, Bukarest, 1978.

13. Tamaș,Ioan, Drept matrimonial catolic, Iaşi „Presa Bună” Verlag, 1994. 